\title{
Using the Internet to Provide Psychodynamic Psychotherapy
}

\author{
Robert Johansson, Ronald J. Frederick and Gerhard Andersson
}

\section{Linköping University Post Print}

\section{Tweet}

N.B.: When citing this work, cite the original article.

Original Publication:

Robert Johansson, Ronald J. Frederick and Gerhard Andersson, Using the Internet to Provide Psychodynamic Psychotherapy, 2013, Psychodynamic Psychiatry, (41), 4, 385-412.

Copyright: Guilford Press

http://www.guilford.com/

Postprint available at: Linköping University Electronic Press

http://urn.kb.se/resolve?urn=urn:nbn:se:liu:diva-100384 


\title{
Using the Internet to Provide Psychodynamic Psychotherapy
}

\author{
Robert Johansson, Ronald J. Frederick, \\ and Gerhard Andersson
}

Abstract: Over the last 15 years, there has been a substantial increase in research and clinical implementations of Internet-delivered, cognitive behavioral therapy (ICBT). Several studies on ICBT have been in the format of guided self-help where a therapist guides the patient throughout the whole treatment. ICBT is typically in the form of self-help material (e.g., text or video) which is provided to a client over the Internet with additional therapist contact by e-mail. ICBT has been shown to be effective for various conditions and, in some studies, has shown to be as effective as face-to-face cognitive behavioral therapy for mild to moderate depression, anxiety disorders, and somatic problems. Recently, the field has expanded to include other orientations including psychodynamic psychotherapy. Currently, there are three randomized controlled trials that have tested the efficacy of psychodynamic psychotherapy delivered in this format. The latest published trial focused on an affect-focused, psychodynamic psychotherapy delivered to a sample of participants with mixed depression and anxiety disorders. This article aims to provide a deeper understanding of the process of providing psychodynamic psychotherapy via the Internet. We will give a detailed description of our latest manual and show how psychotherapeutic work is conducted utilizing this text. Furthermore, we provide examples of dialogue between therapist and client from the online environment. Similarities and differences between psychodynamic psychotherapy delivered over the Internet and in face-to-face formats are discussed.

Robert Johansson, M.Sc., Ph.D., candidate in Clinical Psychology, Department of Behavioural Sciences and Learning, Linköping University, Linköping, Sweden.

Ronald J. Frederick, Ph.D., Licensed Psychologist, Center for Courageous Living, California, United States.

Gerhard Andersson, Ph.D., Professor in Clinical Psychology, Department of Behavioural Sciences and Learning, Linköping University, Linköping, Sweden; Department of Clinical Neuroscience, Psychiatry section, Karolinska Institutet, Stockholm, Sweden.

We would like to thank our co-workers from the study: Martin Björklund, Christoffer Hornborg, Stina Karlsson, Hugo Hesser, Brjánn Ljótsson, and Andréas Rousseau. We would also like to acknowledge the participants in the study for their involvement and helpful comments. 
This article provides a detailed description of the practical work of conducting psychodynamic psychotherapy through the Internet and is a complement to a previously published, randomized controlled trial (Johansson et al., 2013). Our aim here is threefold: 1. To illustrate the practical work of psychodynamic therapy in the form of guided selfhelp through the Internet; 2 . To further describe the treatment manual used in the Johansson et al. (2013) trial; and 3. To discuss similarities and differences between Internet-delivered and face-to-face psychodynamic therapy.

\section{INTERNET-DELIVERED PSYCHOTHERAPY AND GUIDED SELF-HELP}

The idea of providing psychotherapy through the Internet is not new. However, almost all available research has been about Internetdelivered cognitive behavioral therapy (ICBT). There is a large body of research supporting the conclusion that ICBT is effective for various conditions such as depression (Andersson \& Cuijpers, 2009; Johansson \& Andersson, 2012), anxiety disorders (Andrews, Cuijpers, Craske, McEvoy, \& Titov, 2010), and various health-related conditions (Andersson, 2009; Hedman, Ljótsson, \& Lindefors, 2012). Also, there are no indications of differential efficacy compared to face-to-face CBT, with a recent meta-analysis showing guided self-help (a majority of studies being ICBT) to be as effective as face-to-face treatments for mild to moderate depression and anxiety disorders (Cuijpers, Donker, van Straten, Li, \& Andersson, 2010).

Most of the research regarding ICBT has been in the form of guided self-help. This approach consists of providing self-help material with additional therapist support. The self-help material can be presented in any modality (e.g., text, video, audio files, or smartphone application). The therapist can have various responsibilities when providing guided self-help including everything from conducting weekly phone calls that follow a pre-specified flowchart to providing well-thought out interpretations of clients' experiences. The precise role of the therapist in this particular study will be described below. Therapist support in guided self-help can be provided via several mediums such as email, phone, or even by face-to-face contact. In most research on guided self-help treatments, the self-help material has been in the form of text provided via the Internet with guidance being given through 10 to 20 minutes of e-mail contact once a week per client. Despite the advancement of technology in the last decade, most Internet-delivered psy- 
chotherapies have used quite simple technology, what we might call "net-bibliotherapy" with basic therapist support (Paxling et al., 2013; Sánchez-Ortiz, Munro, Startup, Treasure, \& Schmidt, 2011). In this text, the terms guided self-help and Internet-delivered psychotherapy/psychological treatments will be used synonymously.

While typical guided self-help treatments only include about 10 to 20 minutes a week of therapist-client contact, this additional support makes a substantial difference in terms of efficacy (Andersson \& Cuijpers, 2009; Richards \& Richardson, 2012). In addition, while self-help treatments without a therapist can be effective, for example, as in the treatment of depression (Cuijpers et al., 2011), it is a stable finding across studies that the therapist contact improves adherence, reduces dropout rates, and leads to better outcomes.

\section{PSYCHODYNAMIC GUIDED SELF-HELP THROUGH THE INTERNET}

The fact that ICBT is often based on self-help texts raises the question of whether there are other models of psychotherapy that can be derived from self-help texts. While many psychodynamic therapies emphasize transference phenomena that, for obvious reasons, is not compatible with a self-help text (discussed below), there are other aspects of psychodynamic theory that could possibly be the basis for self-help material. Examples of psychodynamic self-help books include Make the Leap by Farrell Silverberg (Silverberg, 2005), Unlearn Your Pain by Howard Schubiner (Schubiner \& Betzold, 2012), Think Like a Shrink by Christ L. Zois (Zois \& Fogarty, 1993), and Living Like You Mean It by Ronald J. Frederick (Frederick, 2009).

Given the research developed in the CBT field and the evidence base for psychodynamic therapy delivered in standard face-to-face format, combined with the availability of psychodynamic, self-help books, the idea of developing guided self-help treatments via the Internet based on these books seemed to us a natural next step. Currently, we are aware of three empirical studies on Internet-based psychodynamic psychotherapy, all of which have been conducted by our own research group. The first two studies were based on the book Make the Leap (Silverberg, 2005), and were randomized controlled trials that included patients with Major Depression (Johansson et al., 2012) and Generalized Anxiety Disorder (Andersson et al., 2012). These two studies were the first to provide evidence that psychodynamic psychotherapy delivered through the Internet could be effective. Third, the most recent 
development, was a treatment study in which an affect-focused, psychodynamic intervention was tested on mixed groups of people with depression and anxiety disorders (Johansson et al., 2013). In that study, the treatment was shown to have a large effect on depression and a moderately large effect on anxiety, when compared to a control group who received nonspecific online support. The affect-focused treatment used could be placed in a class of psychodynamic treatments called Experiential Dynamic Therapies (Osimo \& Stein, 2012). The present article complements the recently published Johansson et al. (2013) controlled trial with a detailed description of the treatment, practical examples, and case excerpts.

\section{EXPERIENTIAL DYNAMIC THERAPY AND AFFECT PHOBIA THERAPY}

As stated above, the treatment described in this article was based on Experiential Dynamic Therapy (EDT), which is an umbrella term for psychotherapies that focus on restructuring defenses, regulating anxiety, and promoting the experience and expression of affect (Osimo \& Stein, 2012). In general, EDTs follow the fundamental structure of psychodynamic psychotherapy as outlined by Malan's "Triangle of Conflict" (Figure 1, to the left), a paradigm that illustrates how defenses and anxieties block the expression of feelings, and the "Triangle of Person" (Figure 1, to the right), which illustrates how this conflict originated in past relationships and gets played out with the therapist and people in the patient's current life (Osimo \& Stein, 2012). The present treatment is based on an experiential dynamic therapy called Affect Phobia Therapy in which the client's avoidance of emotional experience is likened to a phobic response (McCullough et al., 2003). In Affect Phobia Therapy, or APT, the therapist typically clarifies defenses, regulates associated anxiety, and helps the client to observe and experience underlying affects (McCullough et al., 2003). Formally, the treatment includes three main objectives: Defense Restructuring (recognizing and relinquishing maladaptive defenses), Affect Restructuring (desensitization through exposure to conflicted affects), and Self/Other Restructuring (improvement in sense of self and one's relationship with others). The main goal of EDT is to help clients experience and express adaptive feelings that had been previously avoided (McCullough et al., 2003). While this goal is shared among all EDTs, the various techniques, as well as the amount of focus on the transference, varies. 

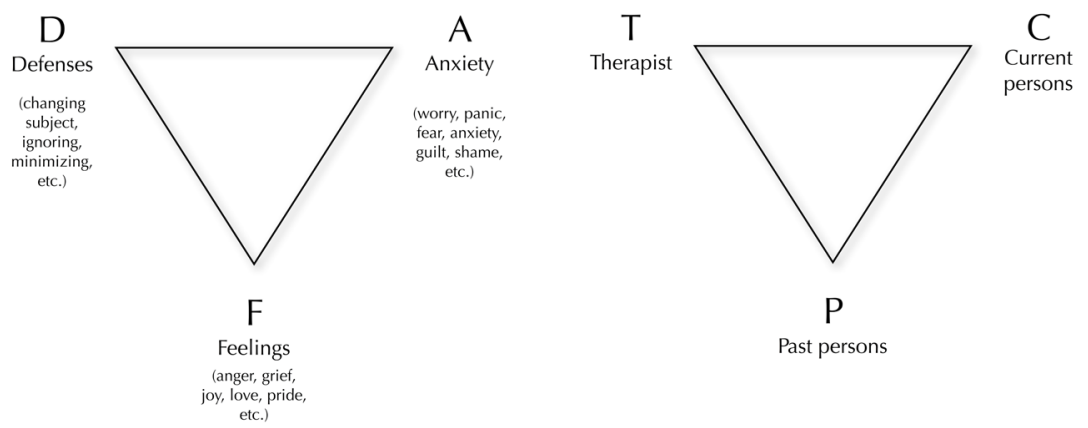

FIGURE 1. Malan's Two Triangles. The two triangles (Malan, 1995) represent what David Malan called "the universal principle of psychodynamic psychotherapy." That is, defenses (D) and anxieties (A) can block the expression of true feelings (F). These patterns began with past persons $(\mathrm{P})$, are maintained with current persons $(\mathrm{C})$, and are often enacted with the therapist $(T)$.

APT conceptualizes conflicts about feelings as "phobias of affects." The therapy is based on the hypothesis that these conflicts / phobias underlie several Axis I and Axis II disorders (McCullough et al., 2003). In line with behavior therapy, systematic desensitization, in the form of exposure to feelings and defense response prevention, is assumed to be fundamental for therapeutic change (McCullough et al., 2003).

\section{ADAPTATION TO THE INTERNET}

The treatment described in this article is based on the book Living Like You Mean It by Ronald J. Frederick (Frederick, 2009). This self-help book is clearly rooted in the experiential dynamic tradition drawing mainly from Affect Phobia Therapy (McCullough et al., 2003) and Accelerated Experiential Dynamic Psychotherapy (Fosha, 2000). To develop a manual appropriate for guided self-help, a series of changes were made to the original text. One important addition was that the Triangle of Conflict was more frequently presented and shared with the client as a model for the treatment. While the model was available in the third chapter of the original text, we incorporated the model throughout the treatment to help explain the rationale for various interventions and to clarify case vignettes. Clearly presenting a model to the client is prominent in CBT treatments (e.g., the cognitive model of depression in CBT; Beck, Rush, Shaw, \& Emery, 1979), and preserving that model is considered fundamental when translating a CBT therapist manual into 
ICBT self-help text. Furthermore, also inspired by ICBT treatments, we added worksheets and homework to the treatment, which is not a prescribed feature of dynamic psychotherapy even if it informally occurs (Stricker, 2006). We hoped that the addition of these exercises would increase the probability that clients would experiment with and practice them, as they were assumed to be essential for making progress in treatment. Finally, some additional clarifying material and case stories were added to the text.

\section{THE ROLE OF THE THERAPIST}

In general, the role of the therapist in our study was to provide assurance that clients progressed through the treatment and completed exercises and homework on time. Tasks included giving feedback on homework, answering any additional questions, providing reference to the text when necessary, encouraging progress, providing support and validation, handling scheduling issues, etc. In most of the trials that we have conducted, the therapist answers any questions within 24 hours during weekdays. While this approach opens up the possibility of fostering frequent contact, most of the contact is typically related to homework. The role of the therapist in this treatment could be said to be similar to that of several ICBT treatments (Andersson, 2009; Andersson et al., 2008). A further description of how the therapist worked in this particular treatment is given in the next section.

\section{THE TREATMENT}

As described above, experiential dynamic therapies (EDT) have the shared goal of trying to access and process repressed feelings and, to that end, make use of various techniques to regulate associated anxiety and dissolve defenses impeding the expression of one's true feelings. In face-to-face EDT, therapists typically direct attention to repressed feelings. For example, the therapist might ask, "What do you notice feeling inside as we begin to look at your experience?" Similarly, it is often the therapist who helps the client become aware of his or her defenses and attend to related anxiety. To this end, she might inquire, "Do you notice that you just changed the subject?" or "It seems that you feel uncomfortable being present with your feelings and move away from them by going into your head. Does that seem right?" Important to note, the role 
of the therapist in the current treatment (described below) does not involve this moment-to-moment interaction. Instead, the self-help material introduces the concept of "emotional mindfulness" (Frederick, 2009), a process through which the client becomes more aware of his feelings and how he relates to them and more able to be present with them. The practice of emotional mindfulness is continued throughout the entire treatment and could be said to be the "heart" of the model described in this article. In other words, the concept of emotional mindfulness translates the EDT tradition into self-help by cultivating one's mindful awareness of and presence with emotions, associated anxiety, and defenses. To be explicit, instead of the therapist encouraging the client to approach feelings, recognize defenses, and regulate anxiety, the client is taught to do these things himself through the use of mindfulness-based tools and techniques. Hence, we believe that the core ingredients of affect phobia therapy are retained in the self-help program developed.

From a psychodynamic perspective, psychiatric problems are understood, in part, as the result of maladaptive defenses used to avoid distress associated with one's feelings. Emotional mindfulness (and the associated skills practiced in the treatment) is offered as an adaptive alternative to being present with one's emotional experience and as a means toward achieving mental health and well-being.

\section{AN OVERALL SUMMARY}

The treatment consisted of five parts-an introduction and orientation to the issue at hand, and then the four-step treatment process: 1. "Becoming Aware of Your Feelings and Defenses," 2. "Taming the Fear," 3. "Feeling it Through," and, 4. "Opening Up." Our treatment introduced the concept of affect phobia early on and encouraged clients to begin to understand their problems from this perspective. After getting a historical understanding of the etiology of their problems, clients were introduced to the concept of emotional mindfulness. A variety of experiential exercises were then shared to help clients become more aware of their feelings, recognize defenses, regulate anxiety, and adaptively experience and express their feelings. The latter techniques were assumed to initiate a restructuring of clients" "Self-Other" experience (i.e., their experience of themselves and others in relation to each other). All modules consisted of about 20 pages of text and included some form of homework (e.g., encouragement to work with and practice the techniques presented in the module). 


\section{Module 1-“To Feel or Not to Feel"}

The initial module was the first out of two in the Preparatory Stage of the treatment process. An introduction to the program was given and the process of working via the Internet and the role of the therapist were explained. A brief summary of all the modules was presented to give an overview of the treatment and the steps in the treatment process were explained. The first module also introduced the concept of affect phobia and encouraged the client to think of their own problems in these terms. A series of case examples illustrating the experience of affect phobia were shared along with a discussion of the specific problem depicted in the vignette. A worksheet, based on the checklist presented on pages 8-10 in the text Living Like You Mean It and titled, "Are You Afraid of Your Feelings?" was presented. Clients were encouraged to check off the behavioral responses to which they identified. Categories of reactions included: "Afraid of feelings in general," "Afraid of being emotionally close or intimate with others," "Uncomfortable with and avoiding sadness or grief," "Afraid of anger or assertiveness," and "Afraid of happiness or pleasure." Examples included "Smiling or laughing when you're actually feeling something else (such as sadness, anger, or fear)," "Always needing to be in control," and "Feeling uncomfortable with prolonged eye contact." The checklist was presented as an illustration of common signs of affect phobia and also served to help the client and therapist get an idea of how comfortable the client was with his/her feelings. The homework for this module was to complete the worksheet and to answer questions about affect phobias. These questions served the purpose of assuring that clients had understood the material.

\section{Module 2-"How the Heck Did I Get This Way?"}

The second and last part of the Preparatory Stage opened with a quote from Maya Angelou, "History, despite its wrenching pain, cannot be unlived, but if faced with courage, need not be lived again" (Frederick, 2009 , p. 23). This quote captures the theme of the chapter. The purpose of this module was to help the client gain a historical understanding of the affect phobias she/he had begun to explore. Making use of the Triangle of Person, this module helped the client identify a "P-C link"an understanding of how these patterns began with past persons $(\mathrm{P})$ 
but are now being enacted with current persons (C). Further, case examples illustrating the influence of history on a person's functioning were discussed. The homework from the module was based on pages 33-35 from the text Living Like You Mean It and involved reflecting on and gaining insight into the emotional environment in which the client grew up. A series of questions were provided to enhance awareness (e.g., "Describe a situation where you or someone else was really angry. What happened? How were feelings that came up taken care of?"). Further homework included a worksheet on "Unwritten Rules," to support awareness of the kinds of direct and indirect messages about emotions that the client had received (e.g., "Message: Getting the silent treatment when you were assertive or angry" and "Implication: Anger is bad and leads to abandonment").

\section{CASE EXAMPLE FROM MODULES 1 TO 4}

The case presented in the following paragraphs is based on an actual participant in the Johansson et al. (2013) trial. Details of the client's story have been removed or changed, to protect the client's identity. "Maria," a single mom in her late 30s, had a history of depression and obsessive worrying. After the first module, she recognized in herself a need to be in control which included avoiding her feelings in general and ruminative thinking, more specifically. In addition, she often felt irritable and depressed and experienced episodes of disinhibition in which she lost her temper. Furthermore, she described detaching from others when feelings arose and having a tendency to dismiss or diminish her accomplishments.

\section{Maria's Work with the First Module}

After the module was completed, Maria wrote to the therapist that she saw herself in some of the examples. She wrote, "I have never thought that my problems had to do with my feelings. The chapter was exciting to read and brought up a lot of thoughts about myself and how I react in various situations." She wrote about her own goals for the treatment: "I want to feel better. I want to stop worrying all the time, and stop running away from relationships. I want to feel good, without having this constant swarm of thoughts around me." 


\section{Therapist Response after the First Week}

The therapist reflected on the fact that Maria had started working quite intensively and affirmed her. Furthermore, the therapist reflected back to Maria some of the themes seen in what she wrote (e.g., that she struggled with a balance between closeness and distance to other people). The therapist asked her to further describe what happens for her in close relationships: "What happens to you when you get close to other people? What are the feelings that you are afraid of then? Is it the closeness or other feelings?" Furthermore, the therapist wrote: "I understand that this process brings up a lot of feelings, especially since you see yourself in the examples from the text. I think you have done a great job this week, in that you have started to explore your feelings." He concluded with "About my comments and questions above-feel free to comment or answer. You could also consider them as an opportunity to reflect further on your work. The next module is activated for you now."

\section{Maria's Work with the Second Module}

At the end of the second week and the completion of the second module, Maria described a series of emotional experiences from her childhood. She reflected on the fact that neither on birthdays nor funerals did her parents show any feelings, and described the emotional climate as "icy and cold." Examples of the consequence of "unwritten rules" included the awareness that she felt guilty for having feelings. However, while Maria described herself as emotionally controlled, she also identified current situations during which she was aware of and could feel a lot of emotion (e.g., in relation to her own children). At the end of the module Maria also wrote about the feelings that came up for her reading and working with the material.

\section{Therapist Answer after the Second Module}

Once again, the therapist affirmed Maria for her work and acknowledged the courage it took to begin to face her feelings in light of her considerable anxiety. The therapist reflected on the fact that several of the stories from Maria's childhood seemed to help make sense of her current need to control her emotions. Furthermore, the therapist asked Maria to describe her experience of feeling guilty for having feelings 
in general, and, more specifically, how that guilt affected her sense of positive feelings toward herself. The therapist also asked if this feeling of guilt was present during the periods of rumination that Maria had described after the first module.

\section{Module 3-“Becoming Aware of Your Feelings"}

Module 3 was the first of two modules on the theme of awareness. This module introduced the concept of emotional mindfulness in regard to one's feelings and explained the treatment rationale for developing this capacity. It is in this module that clients are encouraged to begin to approach their emotional experience. There were two exercises for the client to complete and both focused on attending to one's visceral experience. Both exercises were provided in text form and as recorded sound files (male voice). The first, "Bottom Up versus Top Down" (p. 53 from Living Like You Mean It) involved making use of a body scan. The second, "Awareness Exercise" (pp. 59-62) was about six core emotions (i.e., anger, sadness, happiness, love, fear, and guilt/ shame). For each, the client was encouraged to identify situations in the past that elicited a particular emotion. The following is an excerpt from the exercise: "Love: Remember a tender moment that you shared with a loved one, an experience when someone really came through for you, or a time when you felt particularly loving toward someone in your life. Imagine being in the presence of someone you love, looking at him or her with affection, sharing a warm embrace. What kind of physical sensations do you experience?" All of the exercises focused on the physical correlates of one's feelings. After clients had identified situations and corresponding emotions, they could compare their physical experiences to a list of "common physical manifestations of the six emotions." For example, in the case of love, "Feeling warm inside, as if you were melting," "Feeling of tenderness toward another," and "An inclination to move forward, to embrace and be affectionate." The homework for the third module was to complete the first exercise at least three times and the second at least once. Clients were encouraged to write about their experiences to their therapists.

\section{Maria's Work During the Third Week}

Maria wrote the following after doing the body scan exercise: "I did the exercise several times. I felt my body and my breath. When I repeated the exercise I noticed new things. I think the message I took 
home after this experience was the fact that there are so many reactions in my body that I do not usually notice." After the emotional awareness exercise, Maria wrote: "I also did the second exercise several times. At first, my thoughts were spinning around a lot. Later, I really felt more. I would say that it was easiest for me to experience happiness and love. Shame and guilt really felt like anxiety, or maybe the guilt gave me anxiety?" As a final reflection, she wrote: "I think of all the situations where I probably have felt a lot of feelings but haven't been aware of it. Instead I have had all this control over my feelings all the time. And, most of the time, I wasn't even aware of it! It was really tough to see this. I have cried a lot when thinking about this. A lot of what I show to others is just something like a mask I put on. I have felt really sad about this during the week and it made the work with this chapter really tough."

\section{Therapist Answer to Maria after the Third Week}

The therapist wrote: “Dear Maria, once again I'm impressed by the work you have done. You seem to have learned something about how you deal with feelings. Actually, the piece that you mention about how it is hard to know if a feeling is anxiety or not is very important. A large part of this treatment is about learning to see this difference so that you can learn to handle the anxiety. Thank you very much for what you shared with me. Regarding the sadness you have felt this week, how do you feel after you have cried? Is it relief or does it feel worse? If you want, you can write back to me about it, or you can keep your further reflections for yourself. I have also activated the next module for you now."

\section{Module 4-"Becoming Aware of Your Defenses"}

A quote by Jim Rohn, "The walls we build around us to keep sadness out also keep out the joy," was shared at the start of the fourth module in which awareness of defenses was the primary focus (Frederick, 2009, p. 64). This module gave a thorough introduction to the function and consequences of defenses. The Triangle of Conflict was presented to explain the role of defenses in relation to emotion and associated anxiety. Several case stories were included to illustrate the Triangle of Conflict in action. The client was encouraged to identify his/her own defenses by using a worksheet with a list of "Interpersonal and Intrapersonal 
defenses." Examples of defenses included "Averting your eyes or turning away from someone when feelings start to come to the surface," "Changing the subject," "Not talking at all, shutting down, withdrawing, or going silent" (interpersonal), and "Overthinking issues, getting 'stuck' in your head, not being able to take action" or "Any addictive behavior (alcohol, drugs, food, sex, gambling, shopping, and so on)" (intrapersonal). In addition, another exercise was presented in which the task was to make connections between one's defenses and feelings.

\section{Client Response to Module 4}

In the checklist of interpersonal defenses, Maria, for example, checked "Smiling or laughing when you're actually feeling something else, such as anger or sadness," "Not talking at all, shutting down, withdrawing, or going silent," and "Tensing up your whole body." Examples of intrapersonal defenses that Maria checked were: "Overthinking issues, getting 'stuck' in your head, not being able to take action," "Having to be in control or being overly self-sufficient," and "Keeping yourself busy or distracted."

When Maria wrote about how some of her defenses were connected to feelings, she described how keeping herself occupied or smiling/ laughing were often defenses against feeling sadness. Further defenses related to sadness that she identified included overactivity and overeating. In addition, Maria described how closeness to others was scary to her and that she often observed herself "building walls" around her to avoid interpersonal experiences. She wrote, "I also see now how typically I can talk a lot when I get close to people. When I think of it now, it does probably happen more often with people that I really want to be close to, but maybe do not dare to be really close to."

At the end, she reflected on the work from the module: "I really do have a lot of defenses! Checking them off on the list was a real eyeopener. I have mixed feelings about this, but it really made things clear. I can see myself in so many of the defenses described." She went on to say, "It was really tough to continue after last week. One part of me wanted to flee from all this and during the week I really thought about if I should continue or not. Would I make it? However, I decided to at least read the module for this week and I am happy I did. Even if it was tough to see all my defenses, things are much easier now than at the end of last week." 


\section{The Therapist's Answer after Week 4}

Therapist's response: “Dear Maria, Thank you for what you're writing. I'm happy to learn that you're feeling better now than during last week. I think you have shown a great deal of awareness of your defenses in what you describe. This awareness will certainly be beneficial for you during the treatment, and afterwards. As you write, it is easy to see a lot of defenses in action, when you shine a light in that direction. One very important thing though, defenses are not always 'bad.' They can help us a lot in various situations. However, excessive use of defenses can have negative consequences. To be more aware of your defenses as they happen and not be a 'slave' to them, is one important goal of this treatment. Once again, thank you for sharing your work with me this week. You are now welcome to go on to the next module."

\section{Module 5-“Taming the Fear"}

The fifth module focused on anxiety regulating techniques. The rationale for the techniques, based on the Triangle of Conflict, was explained. For example, when a client approaches an emotional experience $(\mathrm{F})$ and tries to change the way he/she typically responds (D; i.e., letting go of a well-established defense), anxiety (A) is likely to rise. In order to be comfortably present with one's genuine feelings, the ability to regulate one's anxiety is essential. A series of anxiety-regulating "tools" were presented including affect labeling, mindful tracking of experience, diaphragmatic breathing, positive imagery, and stimulation of the vagus nerve.

Homework for module 5 was divided into two sections. In the first part, the client was encouraged to visualize an emotional situation in which he/she had felt anxious. The client could also use the previous audio recordings to achieve this purpose. When visualizing and getting in touch with their distress, clients were instructed to then try each of the anxiety regulating tools at least once. The second part of the homework for module 5 was to choose three of the tools and put them to use in everyday situations. A typical scenario might be to use diaphragmatic breathing during an entire day and really test out this tool in various anxiety-provoking situations. 


\section{Module 6-"Feeling It Through"}

The sixth module, named after the third step of treatment, "Feeling It Through," focused on affective experience. This chapter contained tools to facilitate one's ability to abide with one's emotional experience. The purpose of the tools was to increase the client's capacity to be present with his/her feelings. Examples include, "Practicing Acceptance," "Slowing Down," and "Giving Way." At the end of the module, to further clarify how the components of the treatment fit together, the Triangle of Conflict was presented once again, and the connection between feeling, anxiety, and defenses and the work from the previous modules was reiterated. Homework included applying the tools from the module to an emotionally laden, anxiety inducing situation from the past. Clients were encouraged to let go of their defenses, regulate anxiety, and lean into their emotional experience. Furthermore, they were instructed to repeat this exercise three times during the week in which they worked with the module.

\section{CASE EXAMPLE FROM MODULES 5 TO 8}

"David," a married man in his mid-40s, struggled with anxiety in various situations. In the first module, he identified that his distress was very much related to anger. He noted ways in which he avoided the direct experience of his anger, including: suppression, passive aggression, self-attack, and a "need to please" other people. His primary goal for treatment was to have more intimate relationships with others.

While working with the Naming Tool in the fifth module, David described difficulties in differentiating between some emotions (e.g., sadness from anxiety). He preferred the Mindful Tracking tool with its emphasis on noticing and describing one's physical experience. He also identified the Breathing Tool and the Heart Tool as potential means of regulating his anxiety. He reported a positive experience utilizing the mindfulness based techniques saying, "In social situations that make me anxious, I liked applying the mindfulness tool. I can feel my heart beat and I notice how my body gradually starts to calm down. The naming tool was also OK, I felt somewhat calmer. However, it is still difficult to differentiate some emotions I think." 


\section{Therapist's Response to David Following Module 5}

"Dear David, good work with trying out the tools. It sounds as though you liked to use the mindful tracking tool. Focusing on the body is a good way to start in your efforts to better handle your emotional experience. You write that you have had difficulties differentiating emotions, for example: sadness and anxiety. Can you describe this further? Do you have memories of these emotions from separate occasions? To make sense of what you are experiencing, it might help to look back on the triangle. It seems to me that the 'A corner' (anxiety) and the 'F corner' (e.g., sadness) get confused for you. Does this happen with feelings other than sadness? What do you think about this? About the tools you have started with, I think you can continue with the mindful tracking. It is a good way to start, and then, maybe you can apply the naming tool to label your experiences in the body? Maybe continued practice with both these tools will make it easier to differentiate your experiences?"

\section{David's Work with Module 6}

Subsequent to working with the sixth module, David wrote to his therapist: "I still think it is hard to reach my feelings. I often try to connect with them but do not really find anything. However, I can see that I have defenses going on. The 'thinking' part of me makes it harder sometimes. Regarding the continued work with the previous tools, I still practice the mindful tracking tool and the naming tool. I have also added the staying open tool. I have discovered that when I use that, I feel that I'm really open to my experience, like, staying in that position." Regarding the work with the tools presented in the sixth module, David wrote: "The main problem is that it is hard to reach the feelings, and sometimes when I start to feel something, it is like it goes away so quickly. Why is that? However, when I actually do feel something it is not hard for me to accept the feeling using the acceptance tool. I must say though, that I like this kind of work. I had tried so many things before and this is very much something new. For a thinking guy like me, this thing with feelings is exciting to explore." 


\section{Therapist's Answer after Sixth Week}

The therapist encouraged David to continue the work with the "Staying open tool" and tried to normalize that it can be hard to connect with one's feelings. In addition, the therapist affirmed David's work. He wrote: "David, you are working really hard on this, and you are following the treatment plan. As you have eight modules for ten weeks, maybe this is an opportunity to take some extra time for this one, before continuing onto module 7? For example, why not try out the exercises from the third module again? That could possibly enable you to work from the bottom and go all the way up. If you want, you can also do this in parallel with module 7. I can make it accessible for you, and you can decide how you want to work during the following two weeks. Please let me know what you think."

\section{Module 7-“Opening Up"}

"Opening Up," the last step of the treatment model, concerns affect expression. The purpose of this module was to help clients make use of and communicate their feelings in interpersonal situations. Feelings were shown to impart information, insight, and guidance. A case example illustrated someone utilizing the techniques previously presented to a feeling of anger, the cues she was getting from this feeling, as well as the inherent action tendency to assert herself in a situation where she was neglected by a friend. An exercise was presented to help the client examine and make use of the information contained in feelings associated with a situation in his/her life that the client wanted to change. Furthermore, the module contained a section on how to mindfully communicate one's feelings, wants, needs, and goals. Exercises to practice "mindful communication" in interpersonal situations concluded the chapter.

\section{David's Work with Module 7}

David told the therapist that he wanted to work with module 7 while continuing his work with some of the previous tools. After working on his own for close to two weeks, he wrote to the therapist: "I have really been able to stay open, that is, I have used the staying open tool a lot. Regar- 
ding my defenses, it is like I have dropped a lot of them (for example, with my colleagues at work). These actions, along with the staying open thing, have led me to have several occasions in where I really felt present with my emotions. This kind of experience has been both with my family and at work, both positive and negative feelings." He continued to describe his work with module 7: "I wanted to try to change some things in how my wife and I communicate. It has been really unusual for me to talk about my feelings. To talk about what I want is hard and really not a thing I usually do. It's like I realized that I'm also a part of it. We have had a couple of discussions and small fights during the week. I'm not very comfortable having these discussions with her. In a way, it has always been easier to not say what I want. Just telling her how I felt was new. No miracles happened, but I actually felt proud of myself. I'm definitely curious and will continue this work! Looking forward to the last module."

\section{Therapist's Answer to David after Module 7}

The therapist wrote back to David sharing his pleasure over David's progress. He also encouraged David to give things time and explained that the treatment was about providing tools for continued work both during and after the ten weeks.

\section{Module 8-“Putting It All Together"}

In the last chapter, all the steps of the treatment were reviewed. A few of the cases presented, including an illustration of how their "affect phobias" were worked on over time, were discussed. Clients were encouraged to summarize their own treatment experience. In addition, they were asked to reflect on and write about how they could handle future emotional challenges as well as how to maintain feeling better.

\section{David's Final Work}

David reflected on his entire treatment experience: "The most important thing discovered in this treatment was that I actually do have feelings. To start letting them come out is a great thing. I have also liked this thing with the emotional mindfulness and the mindful tracking. It has helped me to stay present in my daily life. Maybe it is as though I have come closer to myself. I have definitely come closer to other people. Just by acknowledging for myself that I have feelings is a big thing. 
This makes a difference when I am with other people. I notice it at work and at home. Interestingly, I have noticed that I have come closer to my parents as well, even if I didn't do any "practice" with them."

\section{The Therapist's Response to the Ending}

The therapist concluded by reflecting on all the work that David had accomplished and providing a short summary of his view of the treatment. In addition, some formal information on the ending of the research project was shared. The therapist thanked David for giving him the opportunity to follow his experiences and progress. A few informal messages were exchanged as treatment was concluded.

\section{SUMMARY OF THE TREATMENT}

As described above, the main component of the treatment was in the form of written text. The text introduced concepts relevant to the treatment and the fundamental principles upon which the treatment was based (emotional mindfulness and the Triangle of Conflict). In addition, numerous case descriptions helped to bring the concepts to life allowing clients to see their struggles depicted in the experience of others. A set of techniques were introduced and taught to cultivate the skills of emotional mindfulness and help clients be more present with their emotional experience. Clients worked with the various techniques, reflected on their experience, and reported to and discussed with a therapist. Homework was included with the primary purpose of making sure that clients read all of the text and made use of the proposed techniques.

\section{SUMMARY OF THE WORK OF THE THERAPIST}

The techniques provided in the self-help text were assumed to be sufficient for the treatment to progress. This assumption does not mean that we believe that the treatment would be equally effective without a therapist. While the therapist in our study relied mostly on nonspecific supportive techniques to help the client progress through the material, it is still possible to operationalize and describe a few of the interventions used when a therapist provides guidance through the Internet. 
Several supportive ways of intervening included validating and normalizing the client's experience, as well as providing affirmation and encouragement. As seen in the transcripts, the therapist also asked clarifying questions while giving the client the freedom to decide whether to answer. This approach aimed to deepen the client's experience with and understanding gained from working with the material and to promote further reflection. After the third week, the therapist asked about the client's feelings with the goal of helping the client differentiate between defenses and emotional experience (e.g., grief versus regressive crying). There was also some explaining of material (e.g., clarifying the role of defenses), summarizing, and mirroring of what had been written to further highlight the therapeutic activity during the week. The therapist also sought to connect the therapeutic activities to the plan of the entire treatment. As seen in the transcripts, the therapist wrote in a personal way, aiming to build a strong alliance with the client.

In the second half of the treatment, therapeutic responses were most often related to the techniques presented in the text. For example, the therapist clarified the goal of the exercises and used the Triangle of Conflict to further clarify the rationale for the tools being practiced. Questions were asked to deepen the material, not only intellectually, but also emotionally. This aspect of the work is evidenced when the therapist inquired about what particular emotions arose in relation to different memories. As the client was on schedule with the treatment and could benefit from additional practice, the therapist chose to propose additional time working with the tools which, ultimately, proved to be beneficial.

\section{OTHER ISSUES}

\section{The Transference}

In psychodynamic psychotherapy in general, the transference is an important theoretical construct that distinguishes psychodynamic therapies from other approaches (Blagys \& Hilsenroth, 2000; Luborsky $\&$ Crits-Christoph, 1998). In some of the experiential dynamic therapies, transference is viewed as the result of unconscious conflict associated with past relationships that gets triggered with people in one's current life, including the therapist (Davanloo, 2000; Osimo \& Stein, 2012). When unconscious feelings are mobilized in the treatment, anxiety and defenses can be said to have transference implications (e.g., when a defense functions to avoid feelings toward the therapist). As defenses arise in relation to the therapist, the transference provides an oppor- 
tunity in which defenses can be identified (i.e., made conscious) and worked through. Different experiential dynamic therapies rely on the use of the transference to various degrees. Davanloo's ISTDP (Abbass, Town, \& Driessen, 2012; Davanloo, 2000), the most well-researched of EDTs, makes use of the transference to a large degree. Other forms of EDT, like Affect Phobia Therapy (McCullough et al., 2003), and Accelerated Experiential Dynamic Psychotherapy (Fosha, 2000), view transference work as a possibility, but not a necessity.

There are two prominent differences in the provision of psychotherapy in the form of guided self-help through the Internet versus traditional face-to-face therapy. First, the client and the therapist cannot see each other in real time, and in most cases they do not even know how the other person looks or sounds (however, in the Johansson et al., 2013, trial, pictures of the therapists were provided on the homepage describing the study). Second, as most guidance is provided by e-mail, the communication is asynchronous (i.e., the communication does not take place in real time). While these changes to communication likely affect the transference experience, we are not aware of any research that systematically investigates this effect.

Importantly, we do not rule out the possibility of transference phenomena when providing psychotherapy in the format of guided selfhelp, as there is no reason to assume that unconscious feelings toward the therapist might not arise in this setting. However, this treatment program did not include any interventions to address transference phenomena. Similarly, a therapist providing guided self-help does not typically address the relationship and/or defenses against it that might possibly show up in the e-mail communication. While there are no guidelines against such work, our assumption is that the active mechanism of the treatment is in the self-help material and, therefore, communication about the transference is not believed to contribute to the treatment effect.

\section{Supervision}

In the clinical trial described above, eight hours of supervision were included. The supervision was provided by doctoral level psychologist Ronald J. Frederick who authored the original treatment manual (Frederick, 2009). Most of the supervision provided consisted of therapists reporting and describing the work of their clients, followed by a discussion of how to best reinforce the provision of the treatment. Hence, therapists deepened their understanding of the treatment mate- 
rial during supervision as well as how best to support the work of their clients. Supervision followed the principle that the techniques available in the treatment manual were sufficient and therefore no new techniques were introduced or suggested. Instead, when discussing clients who were not proceeding as planned, the focus of supervision was on understanding how a client understood or applied a certain technique, how they might need assistance, or how an additional, alternative explanation of the material might be called for.

In general, adherence is not an issue in guided self-help treatments since a large part of treatment relies on clients reading the treatment manual themselves. However, making sure that clients are reading and understanding the material is an important task for the therapist.

\section{Similarities and Differences}

The description above calls for a discussion of similarities and differences between psychodynamic psychotherapy provided through the Internet and in the traditional face-to-face format.

The model tested emphasizes specific techniques which are assumed to be essential for the effectiveness of the treatment. This emphasis is shared by other experiential dynamic therapies (e.g., Davanloo's ISTDP). As stated by Davanloo (1986): "I believe that dynamic psychotherapy can be not merely effective but uniquely effective, that therapeutic effects are produced by specific rather than nonspecific factors, and that the essential factor is the client's experience of his true feelings about the present and the past" (p. 2). Hence, we do not endorse the idea proposed, for example, by Wampold (2001) that nonspecific factors, socalled "common factors," explain all change in psychotherapy.

While this treatment, as well as several other EDTs, relies on the use of specific techniques, there is a difference in application between the self-help format of treatment and other EDTs. Importantly, the treatment described in this article shares its overall goal with that of many psychodynamic treatments, in general, and with experiential dynamic therapies, in particular, which is: to restructure defenses and anxiety and help clients fully experience their true feelings. To that end, experiential dynamic therapies could also be said to share the same goal of cultivating emotional mindfulness. Therefore, we do not think that the use of self-help techniques is a violation of the overall goal.

The self-help format and the fact that the therapeutic experience is asynchronous likely has implications for working with transference phenomena in such a treatment. As described above, our model does 
not address the transference. This approach highlights our assumption that the use of the transference is not necessary to reach the therapeutic goal. However, we do not rule out the possibility that certain disorders exist in which the use of the transference is essential to treatment. Future research should investigate this matter. Based on our clinical experiences, we believe that some clients benefit therapeutically from the distance created by the guided self-help. Reaching clients who have disorders for which the distance could benefit the treatment is also a topic for further research and investigation.

In the case of affect phobia therapy, the assumed change mechanism is exposure with response prevention (McCullough et al., 2003). This assumption is shared by the present study in which emotional mindfulness was taught as a way of leaning into and being more present with one's feelings without the therapist being present to assist. Some authors have suggested that exposure with response prevention may result in better effects of treatment when clients conduct the exposure by themselves in their natural environment (Röper \& Rachman, 1976; Salkovskis, 1985). Whether this benefit exists in affect phobia therapy and how outcome would be affected is a question for further research. However, it remains possible that self-exposure to one's feelings is at least as effective as exposure to emotion with a therapist present.

As described above, the main difference in the role of the therapist working in a guided self-help format may have to do with therapeutic contact. However, there are also some similarities. For instance, supportive aspects of the therapeutic contact include supportive listening, validation of experience, and answering questions. In addition, one supportive aspect of the case stories presented in the text was to help clients see and understand that people other than themselves also struggle with similar issues. Therefore, the differences should not be overstated. The above transcripts also illustrate examples of interventions that are more toward the "expressive" end on the supportive-expressive continuum as defined by Luborsky (1984). The therapist making sense of the client's experience through the Triangle of Conflict is an example of this kind of intervention. However, these particular interventions were not explicitly a part of this treatment model. The majority of therapist communication could be said to be supportive in nature. We acknowledge research investigating the behavior of the therapist in Internet-based psychological treatments as an important future opportunity. Currently, only a few studies exist in this area (e.g., Paxling et al., 2013; Sánchez-Ortiz et al., 2011).

Our Internet-based treatment used the same conceptual scheme, the Triangle of Conflict, as the original affect phobia manual. However, one difference in approach is that the conceptualization was explicitly 
shared with the client in the self-help treatment. While this kind of intervention is common in CBT, it is probably less typical in psychodynamic treatments. From an experiential dynamic perspective, a psychoeducational intervention could potentially promote a more "left brain" approach on the part of the client (e.g., thinking and reasoning) instead of staying with their "right brain" experience (their emotions), the latter being the main goal of the treatment. However, not all clients are prone to excessive intellectual defenses and this educational work can be helpful. This line of reasoning is similar to the APT manual in which a self-restructuring approach is recommended initially when a client's anxiety is too severe (McCullough et al., 2003). In Intensive Short-Term Dynamic Psychotherapy, another EDT, a so-called "graded format" is used for clients who are too fragile to tolerate a build-up in conflicted affect and associated anxiety (Abbass \& Bechard, 2007; Davanloo, 1990; Whittemore, 1996). Ultimately, both of these approaches aim to build capacity in the client before the traditional therapeutic approach can be followed (i.e., exposure to conflicted feelings). In conclusion, making an explicit formulation in terms of the Triangle of Conflict may also function as a capacity-building intervention.

\section{FUTURE IMPLICATIONS AND CHALLENGES}

Obvious implications of making guided self-help treatments available are the possibility of reaching more clients, both due to the shorter time needed to deliver the treatment as well as accessibility.

The fact that psychodynamic treatments that have been empirically validated in guided self-help format may be equally effective when delivered in face-to-face format has implications for the dissemination of psychodynamic therapies in general. Given that the therapist interventions in this model were mostly supportive, it is possible that a face-toface implementation of the treatment tested may require less therapist training. This factor could allow for easier dissemination of treatment.

We have described here the first attempt to implement an Internetbased psychodynamic treatment based on Experiential Dynamic Therapy. There are many ways to expand this body of research. For instance, while our study was with a mixed sample of clients with depression and anxiety disorders, the model could be used with other specific diagnostic groups such as social phobia, panic disorder, and generalized anxiety disorder. Such treatment could establish a focus on issues that tend to be common for these disorders (e.g., feelings of shame and selfworthiness in social phobia). As mentioned above, another direction for future work is to approach groups of clients with more severe character 
pathology. For example, Borderline Personality Disorder is a condition characterized by low distress tolerance and the use of projective defenses. The guided self-help format could be useful in addressing such pathology allowing affect-phobic patterns of behavior to be addressed while maintaining a clear relational boundary. In addition, exploring transference patterns occurring in an asynchronous format is a possible topic for further research.

As stated by Andersson (2010), one contribution of ICBT to the field of CBT in general, is the possibility of accelerating psychotherapy research. For example, the entire evidence base of exposure-based CBT in the treatment of irritable bowel syndrome was established through the use of ICBT (Ljótsson et al., 2010, 2011). The Internet-based format opens up the possibility of large scale trials with national recruitment, in which a tested protocol can be gradually refined (i.e., by adding or removing components) using repeated trials. The Johansson et al. (2013) study is an example on how these options are also possibilities for the field of psychodynamic research. We believe that we have no reason to assume that the treatment tested in Johansson et al. (2013) would be less effective if delivered as face-to-face therapy. Hence, the trial is an important addition to the evidence base of Affect Phobia Therapy in the treatment of Axis I disorders, and is an example of how the Internetbased format was used to provide the evidence.

\section{CONCLUSION}

In this article, we have described a recent development in the provision of psychodynamic psychotherapy. Our overall aim here has been to provide details on an Internet-based psychodynamic treatment that recently has been proven to be effective in the treatment of depression and anxiety disorders. To that end, we have described our approach to providing psychodynamic based psychotherapy through the Internet. While psychological treatments can be provided through the Internet in various ways, the treatment presented here was given in the format of guided self-help. Details have been provided, both regarding the selfhelp material as well as actual dialogue between client and therapist. The treatment described here was a ten-week psychodynamic psychotherapy rooted in the experiential dynamic therapy tradition that made use of therapeutic techniques in a self-help format. Therapists communicated by e-mail in a supportive fashion with the aim of building a strong alliance and to help clients through the self-help material. The work presented in this article provides the basis for a new approach to providing psychodynamic psychotherapy in the 21st century. 


\section{REFERENCES}

Abbass, A., \& Bechard, D. (2007). Bringing character changes with Davanloo's intensive short-term dynamic psychotherapy. Ad Hoc Bulletin of Short-Term Dynamic Psychotherapy, 11(2), 26-40.

Abbass, A., Town, J., \& Driessen, E. (2012). Intensive short-term dynamic psychotherapy: A systematic review and meta-analysis of outcome research. Harvard Review of Psychiatry, 20(2), 97-108. doi: 10.3109/10673229.2012.677347

Andersson, G. (2009). Using the Internet to provide cognitive behaviour therapy. Behaviour Research and Therapy, 47(3), 175-180. doi: 10.1016/j.brat.2009.01.010

Andersson, G. (2010). The promise and pitfalls of the internet for cognitive behavioral therapy. BMC Medicine, 8, 82. doi: 10.1186/1741-7015-8-82

Andersson, G., Bergström, J., Buhrman, M., Carlbring, P., Holländare, F., Kaldo, V., et al. (2008). Development of a new approach to guided self-help via the Internet: The Swedish experience. Journal of Technology in Human Services, 26(2-4), 161-181. doi: 10.1080/15228830802094627

Andersson, G., \& Cuijpers, P. (2009). Internet-based and other computerized psychological treatments for adult depression: A meta-analysis. Cognitive Behaviour Therapy, 38(4), 196-205. doi: 10.1080/16506070903318960

Andersson, G., Paxling, B., Roch-Norlund, P., Östman, G., Norgren, A., Almlöv, J., et al. (2012). Internet-based psychodynamic vs. cognitive behavioural guided self-help for generalized anxiety disorder: A randomised controlled trial. Psychotherapy and Psychosomatics, 81, 344-355.

Andrews, G., Cuijpers, P., Craske, M. G., McEvoy, P., \& Titov, N. (2010). Computer therapy for the anxiety and depressive disorders is effective, acceptable and practical health care: A meta-analysis. PloS ONE, 5(10), e13196. doi: 10.1371/ journal.pone.0013196

Beck, A. T., Rush, A. J., Shaw, B. F., \& Emery, G. (1979). Cognitive therapy of depression. New York: Guilford.

Blagys, M., \& Hilsenroth, M. (2000). Distinctive features of short-term psychodynamic-interpersonal psychotherapy: A review of the comparative psychotherapy process literature. Clinical Psychology: Science and Practice, 7(2), 167188.

Cuijpers, P., Donker, T., Johansson, R., Mohr, D. C., van Straten, A., \& Andersson, G. (2011). Self-guided psychological treatment for depressive symptoms: A meta-analysis. PLoS ONE, 6, e21274.

Cuijpers, P., Donker, T., van Straten, A., Li, J., \& Andersson, G. (2010). Is guided selfhelp as effective as face-to-face psychotherapy for depression and anxiety disorders? A systematic review and meta-analysis of comparative outcome studies. Psychological Medicine, 40(12), 1943-1957. doi: 10.1017/S0033291710000772

Davanloo, H. (1986). Intensive short-term psychotherapy with highly resistant patients. I. Handling resistance. International Journal of Short-Term Psychotherapy, 1(2), 107-134.

Davanloo, H. (1990). Unlocking the unconscious: Selected papers of Habib Davanloo. New York: Wiley.

Davanloo, H. (2000). Intensive short-term dynamic psychotherapy: Selected papers of Habib Davanloo. Chichester: Wiley.

Fosha, D. (2000). The transforming power of affect: A model for accelerated change. New York: BasicBooks. 
Frederick, R. J. (2009). Living like you mean it: Use the wisdom and power of your emotions to get the life you really want. San Francisco: Jossey-Bass.

Hedman, E., Ljótsson, B., \& Lindefors, N. (2012). Cognitive behavior therapy via the Internet: A systematic review of applications, clinical efficacy and costeffectiveness. Expert Review of Pharmacoeconomics and Outcomes Research, 12(6), 745-764. doi: 10.1586/erp.12.67

Johansson, R., \& Andersson, G. (2012). Internet-based psychological treatments for depression. Expert Review of Neurotherapeutics, 12(7), 861-870. doi: 10.1586/ ern.12.63

Johansson, R., Björklund, M., Hornborg, C., Karlsson, S., Hesser, H., Ljótsson, B., et al. (2013). Affect-focused psychodynamic psychotherapy for depression and anxiety through the Internet: A randomized controlled trial. PeerJ, 1, e102. doi: 10.7717 / peerj.102

Johansson, R., Ekbladh, S., Hebert, A., Lindström, M., Möller, S., Petitt, E., et al. (2012). Psychodynamic guided self-help for adult depression through the internet: A randomised controlled trial. PLoS ONE, 7(5), e38021. doi: 10.1371/ journal.pone.0038021

Ljótsson, B., Falk, L., Vesterlund, A. W., Hedman, E., Lindfors, P., Rück, C., et al. (2010). Internet-delivered exposure and mindfulness based therapy for irritable bowel syndrome-A randomized controlled trial. Behaviour Research and Therapy, 48(6), 531-539. doi: 10.1016/j.brat.2010.03.003

Ljótsson, B., Hedman, E., Andersson, E., Hesser, H., Lindfors, P., Hursti, T., et al. (2011). Internet-delivered exposure-based treatment vs. stress management for irritable bowel syndrome: A randomized trial. The American Journal of Gastroenterology, 106(8), 1481-1491. doi: 10.1038/ajg.2011.139

Luborsky, L. (1984). Principles of psychoanalytic psychotherapy: A manual for supportiveexpressive treatment (Vol. 2000). New York: Basic Books.

Luborsky, L., \& Crits-Christoph, P. (1998). Understanding transference: The core conflictual relationship theme method (2nd ed.). Washington, DC: American Psychological Association.

Malan, D. (1995). Individual psychotherapy and the science of psychodynamics (2nd ed.). Oxford: Butterworth-Heinemann.

McCullough, L., Kuhn, N., Andrews, S., Kaplan, A., Wolf, J., \& Hurley, C. L. (2003). Treating affect phobia: A manual for short-term dynamic psychotherapy. New York: Guilford.

Osimo, F., \& Stein, M. J. (2012). Theory and practice of experiential dynamic psychotherapy. London: Karnac.

Paxling, B., Lundgren, S., Norman, A., Almlöv, J., Carlbring, P., Cuijpers, P., \& Andersson, G. (2013). Therapist behaviours in internet-delivered cognitive behaviour therapy: Analyses of e-mail correspondence in the treatment of generalized anxiety disorder. Behavioural and Cognitive Psychotherapy, 41(3), 280-289. doi: 10.1017/S1352465812000240

Richards, D., \& Richardson, T. (2012). Computer-based psychological treatments for depression: A systematic review and meta-analysis. Clinical Psychology Review, 32(4), 329-342. doi: 10.1016/j.cpr.2012.02.004

Röper, G., \& Rachman, S. (1976). Obsessional-compulsive checking: Experimental replication and development. Behaviour Research and Therapy, 14(1), 25-32.

Salkovskis, P. M. (1985). Obsessional-compulsive problems: A cognitive-behavioural analysis. Behaviour Research and Therapy, 23(5), 571-583. 


\section{JOHANSSON ET AL.}

Sánchez-Ortiz, V. C., Munro, C., Startup, H., Treasure, J., \& Schmidt, U. (2011). The role of email guidance in internet-based cognitive-behavioural self-care treatment for bulimia nervosa. European Eating Disorders Review: The Journal of the Eating Disorders Association, 19(4), 342-348. doi: 10.1002/erv.1074

Schubiner, H., \& Betzold, M. (2012). Unlearn your pain: A 28-day process to reprogram your brain (p. 322). Pleasant Ridge, MI: Mind Body Publishing.

Silverberg, F. (2005). Make the leap: A practical guide to breaking the patterns that hold you back. New York: Marlowe and Company.

Stricker, G. (2006). Using homework in psychodynamic psychotherapy. Journal of Psychotherapy Integration, 16(2), 219-237.

Wampold, B. E. (2001). The great psychotherapy debate: Models, methods, and gindings (p. 263). Mahwah, NJ: Erlbaum.

Whittemore, J. W. (1996). Paving the royal road: An overview of conceptual and technical features in the graded format of Davanloo's intensive short-term dynamic psychotherapy. International Journal of Short-Term Psychotherapy, 11(1), 21-39.

Zois, C., \& Fogarty, P. (1993). Think like a shrink: Solve your problems yourself with short term therapy techniques. New York: Grand Central Publishing.

Robert Johansson

Department of Behavioural Sciences and Learning

Linköping University

SE-58183 Linköping, Sweden

robert.johansson@liu.se 